\title{
Indução do trabalho de parto
}

\author{
Aline Cristina de Poli*, Mariana Torreglosa Ruiz**, Zaida Aurora Geraldes Sperli Soler, D.Sc.***
}

*Enfermeira, Aluna do curso de especialização em Enfermagem Obstétrica da Faculdade de Medicina de São José do Rio Preto (FAMERP), ${ }^{*}$ Enfermeira obstétrica, aluna do curso de pós-graduação nivel mestrado em Enfermagem em Saúde Pública, Escola de Enfermagem de Ribeirão Preto, Universidade de São Paulo (EERP-USP), ${ }^{* * *}$ Obstetriz, enfermeira, livre-docente em Enfermagem, docente e orientadora da graduação e da pós-graduação da Faculdade de Medicina de São José do Rio Preto (FAMERP)

\section{Resumo}

A indução do parto consiste na iniciação intencional e artificial do trabalho de parto antes do desencadeamento espontâneo, com o propósito da expulsão da unidade feto-placentária, e apresenta benefícios inquestionáveis quando bem indicadas. Neste artigo faz-se uma revisão da literatura sobre indução do trabalho de parto, enfocando incidência; indicaçóes; contra-indicações relativas e absolutas; possíveis riscos; métodos de avaliação e técnicas mecânicas e farmacológicas mais utilizadas, apresentando dados quanto à eficácia e efeitos colaterais.

Palavras-chave: indução do trabalho de parto, misoprostol e ocitocina.

\section{Abstract \\ Induction of labor}

Labor induction is the intentional and artificial initiation of labor before its spontaneous onset, and with the purpose of expelling the feto-placental unit. Its benefits are unquestionable, when it is used with proper obstetric indication. This study is a literature review regarding induction of labor. The main focuses were: incidence; indications; relative and absolute contraindications; possible risks; evaluation methods, and most widely used mechanical and pharmacological techniques. We present data concerning its efficacy and collateral effects.

Key-words: induction of labor, misoprostol and oxytocin.

\section{Resumen \\ Inducción del trabajo de parto}

La inducción del parto consiste en la iniciación intencional y artificial del trabajo de parto antes del desencadenamiento espontáneo del mismo, y tiene el propósito de expulsar a la unidad feto-placentaria. La inducción presenta beneficios 
incuestionables cuando está bien indicada. En este artículo hicimos una revisión de la literatura sobre inducción del trabajo de parto. Enfocamos: incidencia; indicaciones; contraindicaciones relativas y absolutas; posibles riesgos; métodos de evaluación, y técnicas mecánicas y farmacológicas más utilizadas. Se presentan datos en cuanto a eficacia y efectos colaterales.

Palabras-clave: inducción del trabajo de parto, misoprostol y oxitocina.

\section{Introdução}

A indução do trabalho de parto tem por objetivo desencadear ou acelerar as contraçóes uterinas, de acordo com a dilatação esperada a cada etapa do período de dilataçáo do parto, por meio do uso de métodos artificiais o trabalho de parto, com o propósito de expulsar feto e placenta. Este procedimento terapêutico é considerado importante quando há benefícios para a mãe e feto, principalmente quando possibilita a realização do parto vaginal $[1,2]$.

A indução do trabalho de parto pode ser definida como iniciação intencional do trabalho de parto antes do desencadeamento espontâneo, com o propósito da expulsão da unidade feto placentária [1-3]. O índice de utilização de métodos de indução varia de acordo com o local e a conduta do médico obstetra e geralmente é maior que $20 \%$, sendo considerado um dos procedimentos mais utilizados em Obstetrícia para a resolutividade do parto [3,4].

A incidência de partos induzidos é bastante variável, enquanto nos países europeus não ultrapassa 5\%, nos Estados Unidos aproximadamente 20\% das mulheres recebem ocitocina durante o trabalho de parto, 13\% dessas com a finalidade de indução, o que significa que nascem anualmente mais de 800 mil crianças que tiveram os partos induzidos [5]. Alguns estudiosos verificaram que entre os anos de 1990 a 2005, a incidência da indução eletiva do trabalho de parto, isto é, sem razôes clínicas ou obstétricas que a justificassem, duplicaram de aproximadamente $10 \%$ em 1990 para $21 \%$ em 2005 [6-8].

Desta forma, o termo “indução do parto" é utilizado para todos os procedimentos que podem provocar contraçóes uterinas e desencadear conseqüentemente o trabalho de parto em mulheres com mais de 22 semanas de idade gestacional [9]. Portanto, difere da estimulação das contraçôes uterinas, nas quais o emprego, geralmente da ocitocina, pretende corrigir quadros de hipoligossistolia em partos iniciados espontaneamente. A indução é uma intervenção ativa, com potenciais riscos para a mãe e para o feto [5].

\section{Indicaçóes e contra-indicaçóes para induçáo do trabalho de parto}

A indução do trabalho de parto tem mérito inquestionável quando os benefícios do parto imediato superam os riscos de manter a gestação. Entretanto, os fatores mais comumente citados para a induçáo eletiva são desejo da paciente e conveniência do obstetra [8].

As indicações mais comuns para indução do trabalho de parto estão relacionadas a fatores maternos e obstétricos. Dentre as condições maternas são citadas: síndromes hipertensivas, nefropatias crônicas, diabetes mellitus e colagenoses. As principais indicações obstétricas para indução são em ordem de freqüência: gestação pós termo ou prolongada (acima de 42 semanas gestacionais), corioamniorrexe prematura, óbito fetal, isoimunização e restrição de crescimento intra-uterino [1].

Os autores destacam que existem contraindicações absolutas e relativas para a indução do trabalho de parto. Quanto às contra-indicaçóes absolutas são destacadas: desproporção céfalo-pélvica, apresentaçóes viciosas, descolamento prematuro de placenta normo-inserida, placenta prévia centrototal e presença de sofrimento fetal agudo com cérvice desfavorável [1]. Dentre as contra-indicaçôes relativas destacam-se: cicatriz de cesárea prévia ou de leiomiomectomia, prenhez múltipla, poliidrâmnio, grandes multíparas, isto é com cinco ou mais partos anteriores, insuficiência placentária e em apresentaçóes pélvicas $[1,5]$.

Embora seja uma contra-indicação relativa, a induçáo do parto na existência de antecedente de cicatriz uterina anterior, como na cesárea, apresenta risco 6 vezes maior de rotura uterina, o que demanda vigilância, monitorizaçáo e cuidados durante o trabalho de parto [5].

A indução do parto está associada a maiores riscos de prematuridade, baixo peso ao nascer, distocias, aumento do índice de partos fórceps e cesáreas e excessiva atividade uterina com alteraçóes na freqüência cardíaca fetal $[2,3,7,8]$. O aumento da idade gestacional aumenta o risco para o parto cesá- 
rea, independentemente se o trabalho de parto foi induzido ou evoluiu espontaneamente. A indução do trabalho de parto, portanto, pode náo aumentar o risco de parto cesárea quando comparado ao manejo expectante (trabalho de parto espontâneo), mas sim, estar associado à idade gestacional [10].

\section{Métodos de avaliaçáo para induçáo}

\section{Status cervical}

Um dos mais importantes preditores do sucesso na indução do trabalho de parto é a condição da cérvice. Em 1931, Calkins et al. avaliaram 5700 mulheres em trabalho de parto e sugeriram que a intensidade das contraçóes e consistência uterina, como espessura da parede uterina; comprimento do canal de parto poderiam ser classificados em uma escala de 1 a 5 e, esses fatores provavelmente fariam a previsão da duração do trabalho de parto. Este foi o primeiro sistema de escore cervical sugerido [2].

\section{Índice de Bishop e outros métodos de avaliação: uso de ultrassom transvaginal e marcadores bioquímicos}

Em 1964, Bishop elaborou um índice prognóstico para a induçáo do parto. A extensa bibliografia que decorre de seu emprego, por mais de 30 anos, documenta a importância que ele tem na seleção dos casos favoráveis à induçáo do trabalho de parto [5]. $\mathrm{O}$ índice de Bishop consiste em cinco componentes: dilatação cervical, esvaecimento, consistência uterina, descida fetal e posiçáo do colo e a condição da cérvice prediz o sucesso do trabalho de parto antes do início da indução, tanto em nulíparas quanto em multíparas. Utiliza-se o índice de Bishop para avaliar se a cérvice é favorável antes do início da indução. Assim, é consenso de que índice igual ou maior do que 6 associa-se com êxito em mais de 90\% das induçôes. Por outro lado, pontuaçóes menores servem para contra-indicar induçóes ou referendar o emprego de técnica para o amadurecimento cervical antecedente $[1,2,5,11,12]$.

Mais recentemente, em estudo realizado por Rozenbert et al., com exames de 266 grávidas antes de proceder à indução, chegou-se à conclusão que o índice de Bishop é melhor do que a avaliação ecográfica do comprimento do colo na predição do intervalo indução-parto e da ocorrência de um parto vaginal [13]. Tais consideraçóes revelam que o índice de Bishop ainda apresenta-se como melhor alternativa para avaliação da maturação cervical, apresentando baixo custo e alta acurácia [14].

$\mathrm{O}$ uso do ultrassom transvaginal e a avaliação de marcadores bioquímicos, como a fibronectina fetal para predizer o sucesso da induçáo do trabalho de parto náo apresentaram resultados superiores quando comparados à avaliação através do índice de Bishop [2,12].

\section{Técnicas de indução}

Antes de discorrer sobre as técnicas propriamente ditas, é relevante que se façam algumas consideraçốes no que diz respeito às condiçôes para sua aplicabilidade. O Colégio Americano de Obstetras e Ginecologistas (ACOG) e a Sociedade Canadense de Obstetras e Ginecologistas (CSOG) consideram que no parto induzido é no mínimo recomendável o emprego da monitorização eletrônica [5].

Deste modo, o ambiente hospitalar deve estar adequadamente instrumentalizado ao se empregar drogas ocitócicas, por via intravenosa, com uso de bomba de infusão. Também devem estar disponíveis drogas uterolíticas e anestésicos, bem como material para a prática do fórcipe e da cesárea de urgência. A evoluçáo do trabalho de parto deve ser documentada em partograma e tanto as condiçôes clínicas maternas, quanto a vitalidade do concepto devem estar asseguradas pela prática de metodologia propedêutica adequada [1,5].

Existe uma variedade de métodos para indução do parto, que podem ser divididos em farmacológicos e mecânicos. A grande diferença é que os agentes farmacológicos freqüentemente realizam o preparo da cérvice e induzem o trabalho de parto, enquanto que os agentes mecânicos promovem o preparo cervical sem induzir o trabalho de parto, necessitando algumas vezes, do uso de um segundo agente, como a ocitocina. Esses métodos são utilizados para pacientes com índice de Bishop desfavorável [6].

\section{Métodos mecânicos de indução}

Os métodos mecânicos para o preparo cervical têm sido utilizados desde a época de Hipócrates. Entretanto, nas últimas décadas, vem sendo substituídos pelos métodos de indução farmacológicos [6]. Possuem baixo custo e redução de alguns efeitos colaterais, citados como vantagens que os métodos 
mecânicos apresentam sobre os métodos farmacológicos [9].

\section{Dilatadores osmóticos}

São considerados dilatadores osmóticos os compostos com materiais hidrofílicos, que absorvem água e gradualmente esvaecem e dilatam a cérvice. Os dois materiais mais comumente utilizados são: compostos estéreis de algas marinhas (Laminaria japonica ou Laminaria digitata) e materiais hidrofílicos sintéticos (Lamicel e Dilapan) [6].

As algas possuem ação física e farmacológica, absorvendo água do estroma cervical, que causa a desidratação do estroma, esvaecendo a cérvice e liberando prostaglandinas. A ação dos materiais sintéticos é primariamente mecânica causando a despolimerização das fibras colágenas e aumento à sensibilidade à prostaglandinas E2 (PGE2) $[6,12]$.

Os dilatadores osmóticos ganharam popularidade após o aborto ser legalizado nos Estados Unidos, na década de 1970, mostrando-se seguros quando utilizados para esse fim e desde então vêm sendo utilizados para preparo cervical. Embora, apresentem reduçáo no índice de partos cesárea, seu uso tem diminuído devido a relatos de infecção (associado com infecção por clostridium e gangrena) [6].

\section{Sonda Foley}

Este método mecânico continua sendo bastante utilizado, explicando-se que o uso da sonda Foley na indução do trabalho de parto age por meio de ação mecânica, causando a liberação de prostaglandinas. A liberação de prostaglandinas seria conseqüência da separaçáo do cório da decídua [9].

Achados científicos relatam que o primeiro uso do balão da Foley para dilatação cervical foi descrito por Krauss em 1853 [6]. O método apresenta as seguintes vantagens: baixo custo, estabilidade em temperatura ambiente e baixa incidência de anormalidades das contraçôes uterinas, como taquissistolia e hiperestimulação uterina [6].

É interessante destacar que a sonda promove o amadurecimento cervical, entretanto às vezes se faz necessário associar o uso de ocitocina, que é a responsável pelo incremento da contração uterina. Desta maneira, a associação de sonda Foley e ocitocina pode ser táo efetiva quanto o uso do misoprostol para a indução do parto [9].

\section{Métodos farmacológicos}

Considera-se a indução através de métodos farmacológicos quando se utiliza a infusão de ocitocina ou a administração de prostaglandinas durante o trabalho de parto.

Misoprostol

O misoprostol é uma prostaglandina E1, metil-análoga, que foi originariamente sintetizada e comercializada com o objetivo de diminuir os efeitos colaterais e aumentar o tempo de ação da PGE1 natural, no tratamento de síndromes dispépticas do trato gastrointestinal, como gastrite e úlcera péptica $[13,14]$.

Embora não previsto inicialmente como parte de suas indicaçóes, o misoprostol ganhou popularidade em obstetrícia, descrevendo-se inicialmente o uso para interrupção das gestaçóes de primeiro trimestre, já em 1986, e, logo em seguida, para interrupção de gravidez com feto morto [9].

$\mathrm{O}$ misoprostol tem indicação preferencial para induzir o trabalho de parto em colos com índice de Bishop desfavorável. Sua ação primordial é despolimerizar as fibras colágenas e aumentar a atividade uterina [1]. Além desta açáo, o misoprostol atua sobre a matriz extracelular, com dissolução das fibras colágenas, aumento do ácido hialurônico e aumento do conteúdo de água da cérvice. Também, relaxa o músculo liso da cérvice e facilita a dilatação, ao mesmo tempo em que permite o acréscimo do cálcio intracelular, promovendo contração uterina. Todos estes mecanismos permitem o progressivo esvaecimento e dilatação cervical, concomitante ao aumento da atividade uterina, o que garante na maior parte dos casos uma induçáo bem sucedida do trabalho de parto [9].

O misoprostol apresenta importantes vantagens para o uso na clínica obstétrica. Seu custo é inferior ao de qualquer outra prostaglandina; tem tempo de vida meia-vida alargado; é de fácil administração e não requer refrigeraçáo para sua estocagem. Segundo as pesquisas, constatou-se que o misoprostol é tão eficiente ou mais que as outras prostaglandinas e mais eficaz que a ocitocina na indução do parto com colo não maduro [9].

Em relação à dose utilizada na indução do parto, devido ao aumento da incidência de efeitos uterotônicos com altas doses de misoprostrol, as recomendações feitas pelo Colégio Americano de Obstetras e Ginecologistas é de que se utilize 25 mcg com intervalo de 03 a 06 horas [4]. 
O misoprostrol pode ser administrado via oral ou via vaginal, entretanto, a via de administração ainda é alvo de controvérsias. Os achados na literatura apontam que o uso vaginal mostrou resultados eficazes, mas apresenta altos índices de hiperestimulação uterina, que podem ser minimizados simplesmente através da remoção do agente da vagina. Quando utilizado via oral, apresenta como principal efeito colateral, náuseas e vômitos, podendo não alcançar os resultados esperados. Quando administrado via endovenosa, foram observadas as seguintes desvantagens: hiperestimulação uterina e hipertemia, com difícil diferenciação da causa - se devido à PGE2 ou corioamnionite [14].

O uso do misoprostol via oral oferece teoricamente uma única vantagem, evita as avaliações vaginais e aumenta a aceitação do tratamento pela paciente [15].

Em mulheres com cicatriz uterina com cérvice desfavorável que requerem indução do trabalho de parto, a administração via vaginal de misoprostrol tem se mostrado um método seguro e eficaz. Alguns estudos com amostras reduzidas sugerem que o uso do misoprostrol em mulheres com cicatriz prévia de cesárea, aumenta a freqüência de rotura da cicatriz uterina, deiscência de cicatriz e rotura uterina. Portanto, seu uso náo é recomendado nestes casos, o que ainda é controverso na prática obstétrica [4]. Em relação à eficácia, os resultados em relação ao intervalo entre o trabalho de parto e parto; resultados neonatais e efeitos colaterais maternos foram semelhantes. Portanto, o misoprostrol apresenta-se como alternativa viável, segura, econômica e efetiva [16-18].

\section{Ocitocina}

A ocitocina é sintetizada nos núcleos paraventricular e supraótico do hipotálamo, em um largo precursor peptídeo que passa pelo processo de clivagem. Após a clivagem a ocitocina é estocada em associação com outra proteína nas glândulas de armazenagem nos nervos terminais localizados na pituitária posterior. $\mathrm{O}$ estímulo para liberação da ocitocina inclui estímulo sensorial da cérvice, vagina e mama. Em resposta, a ocitocina é liberada no plasma [19].

As propriedades contráteis da ocitocina no miométrio foram descritas pela primeira vez em 1906. Extraída da pituitária posterior foi inicialmente utilizada na prática clínica para o tratamento da hemorragia puerperal e após, observou-se ação na indução do trabalho de parto. A ocitocina dificilmente é administrada por via oral, pois é rapidamente metabolizada pelo trato gastrointestinal. Assim, a via de escolha geralmente é endovenosa contínua, para indução e condução do trabalho de parto, sendo o tempo para a resposta uterina de 3 a 5 minutos e com 40 minutos o nível de estabilização é alcançado. A ocitocina é rapidamente metabolizada por muitas enzimas incluindo peptidases no rim e a ocitocinase e é secretada pela placenta. Após a inativaçáo por essas enzimas, os metabólitos da ocitocina são excretados na urina [19].

A dose adequada e máxima é obtida quando o útero apresenta resposta à infusão de ocitocina, embora vários autores difundam que a dose máxima é de $40 \mathrm{um} / \mathrm{min}$. Entretanto, quando realizados estudos com altas dosagens de ocitocina, nenhum mostrou aumento da incidência de hemorragia pósparto ou resultados perinatais ruins [20]. A ação da ocitocina é dependente da presença de estrógenos que induzem aumento dos receptores para ocitocina no miométrio e apresentam níveis séricos tanto mais elevados, quanto mais avançada a idade gestacional. Em decorrência disto, a melhor resposta à ocitocina ocorre nas últimas semanas de gestação $[9,13,19]$.

Os principais efeitos colaterais da ocitocina incluem: hiperestimulação uterina (presença de mais que 5 contraçóes em 10 minutos; contraçóes com duração de 2 minutos, ou contrações com duração normal que ocorrem em intervalos menores que 1 minuto entre uma ou outra contração, com ou sem alteraçóes na freqüência cardíaca fetal); taquissistolia (hiperestimulação uterina sem alteraçóes na freqüência cardíaca fetal); hiponatremia (possui estrutura similar à vasopressina (hormônio antidiurético); assim, quando a ocitocina é infundida em altas doses, há reação potencial de reatividade cruzada com os receptores da vasopressina localizados no rim); hipotensão (causada pela infusão em bollus) [19].

\section{Conclusão}

A indução do parto apresenta benefícios inquestionáveis, para máe e para o feto, quando bem indicada. Para isso é necessário uma avaliação adequada das mulheres que necessitam da sua utilização, considerando os dados gestacionais e avaliação da cérvice. A partir da avaliação inicial, escolhe-se o método mais apropriado e seguro para indução do parto. Então, é necessário conhecer a eficácia e pos- 
síveis efeitos colaterais de cada método e utilizá-los segundo a relação risco-benefício.

\section{Referências}

1. Duarte G, Cunha SP, Mauad Filho F, Nogueira AA, Berezowski AT, Rodrigues R, et al. Protocolo de condutas em gestação de alto risco. Ribeirão Preto: Funpec; 2003.

2. Crane JM. Factors predicting labor induction success: a critical analysis. Clinical Obstet Gynecol 2006;49(3):573-84.

3. Crane JM, Butler B, Young DC, Hannah ME. Misoprostrol compared with prostaglandin E2 for labour induction in women at term with intact membranes and unfavorable cervix: a systematic review. BJOG 2006;113:1366-76.

4. Wing DA, Gaffaney CAL. Vaginal misoprostrol administration for cervical ripening and labor induction. Clinical Obstet Gynecol 2006;49(3):627-41.

5. Neme B. Obstetrícia básica. 3a ed. São Paulo: Sarvier; 2005.

6. Gelber S, Sciscione A. Mechanical methods of cervical ripening and labor induction. Clinical Obstet Gynecol 2006;49(3):642-57.

7. Lin MG, Rouse DJ. What is a failed labor induction? Clinical Obstet Gynecol 2006;49(3):585-93.

8. Moore LE, Rayburn WF. Elective induction of labour. Clinical Obstet Gynecol 2006;49(3):698-704.

9. Moraes Filho OB, Cecatti JG, Feitosa FEL. Métodos para induçáo do parto. RBGO 2005;27(8):493-500.

10. Caughey AB, Nicholson JM, Cheng YW, Lyell DJ, Washington AE. Induction of labor and cesarean delivery by gestational age. Am J Obstet Gynecol 2006;195:700-5.

11. Rosenberg P, Chevret S, Chastang C. Comparison of digital and ultrassonographic examination of the cervix in predicting the length of induced labor time interval from induction to delivery in women with a low Bishop score. BJOG 2005;112:192-96.

12. Baacke KA, Edwards RK. Preinduction cervical assessment. Clinical Obstet Gynecol 2006;49(3):564-72.

13. Brasil. Ministério da Saúde. Secretaria de Políticas de Saúde. Área técnica de Saúde da Mulher. Parto, aborto e puerpério: assistência humanizada à mulher. Brasília: Ministério da Saúde; 2001.

14. Keirse MJNC. Natural prostaglandins for induction of labor and preinduction cervical ripening. Clinical Obstet Gynecol 2006;49(3):609-26.

15. Mozurkewich E. Prelabor rupture of membranes at term: induction techniques. Clinical Obstet Gynecol 2006;49(3):672-83.

16. Bartusevicius A, Barcaite E, Krikstolaitis R, Gintautas V, Nadisauskiene R. Sublingual compared with vaginal misoprostrol for labour induction at term: a randomised controlled trial. BJOG 2006;113:1431-37.

17. Haghighi L. Intravaginal misoprostrol in preterm premature rupture of membranes with low Bishop scores. Int J Gynecol Obstet 2006;94:121-2.

18. Zeteroglu S, Engin-Ustun Y, Ustun Y, Guvercinçi M, Sahin G, Kamaci M. A prospective randomized study comparing misoprostrol and oxytocin for premature rupture of membranes at term. J Matern-Fetal Neonat Med 2006;19(5):283-7.

19. Smith J, Merrill DC. Oxytocin for induction of labor. Clinical Obstet Gynecol 2006;49(3):594-608. 